\title{
The delicate topic of progress
}

\section{Diana Epstein ${ }^{1} \cdot$ Angelos Kolias $^{2} \cdot$ Tiit Mathiesen $^{3,4,5}$}

Received: 7 June 2019 / Accepted: 8 June 2019 / Published online: 26 June 2019

(C) Springer-Verlag GmbH Austria, part of Springer Nature 2019

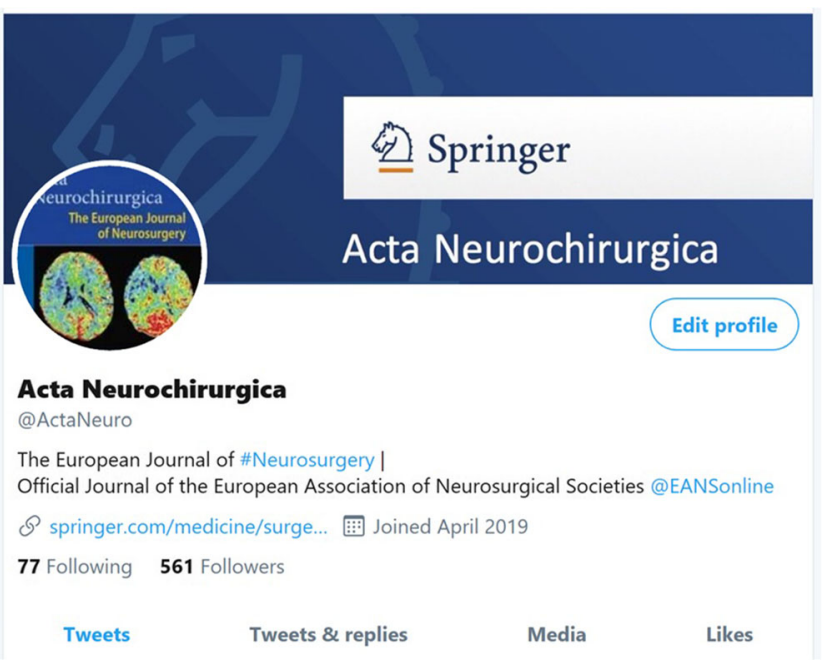

Fig. 1 Screenshot of the @ ActaNeuro Twitter account homepage

"without continual growth and progress, such words as improvement, achievement and success have no meaning" Benjamin Franklin

The landscape of scientific communication is changing rapidly. It is a challenge to improve flexible, rapid communication without compromising scientific quality and accountability. This editorial is a statement to the progress made with the journal during the past few months.

Diana Epstein

acta_neuro@di-ep.com

1 Di-Ep Biomedical Editorial Services Ltd, PO Box 5617, Glasgow, Scotland G77-9EH, UK

2 Division of Neurosurgery, Department of Clinical Neurosciences, Addenbrooke's Hospital, University of Cambridge, Cambridge, UK

3 University of Copenhagen, Copenhagen, Denmark

4 Rigshospitalet, Copenhagen, Denmark

5 Karolinska Intitutet, Stockholm, Sweden
Social media has a growing role in the dissemination of new scientific findings, and alternative metrics have gained importance as complementary to traditional citation metrics, with several publishers tracking both metrics for individual articles [1]. Acta Neurochirurgica has had a Twitter presence via the journal's editorial office account (@diepbiomed) for the past two years, but we are pleased to advise that we now have a dedicated team of Social Media editors. The journal's social media presence is important not only to disseminate our authors' work but also enhance education and foster collaborations between neurosurgeons by virtue of its interactive and immediate nature.

The team of Social Media editors comprises Angelos Kolias (lead, based at Cambridge, UK_—ag_kolias), Alexander Alamri (London, UK-@ AlexAlamri), Claire Karekezi (Kigali, Rwanda-@ @lairekarekezi), Laura Lippa (Siena, Italy_@ @sparklingcsf), Katrin Rabiei (Gothenburg, Sweden_—DrKatrin_Rabiei), and Victor Staartjes (Zurich, Switzerland-@staartjesneuro). Their role is to curate new content and disseminate new papers via the @ ActaNeuro account and their personal accounts, in liaison with the Editorin-Chief and managing editor (Figs. 1, 2, 3, and 4).

The @ ActaNeuro Twitter account went live on the 13 April 2019-with a mix of \#EditorsChoice, \#OnlineFirst, \#OpenAccess, \#HighlyCited, and regular papers.

Since then, 94 Tweets have been sent from the account that has earned 205,000 impressions (as of 5 June 2019). This means that @ ActaNeuro Tweets have been seen by Twitter users 205,000 times in total. As we always include a link to the article on the website, the Tweets have generated 1900 link clicks and 687 retweets. The former underlines the value to the journal and authors in terms of an additional avenue for dissemination of research findings. The latter is also relevant, as it shows that Twitter users are engaging with the @ ActaNeuro account. In total, the account now has 561 followers. This is excellent given how new the account is. We would encourage authors who have a Twitter account to add their Twitter handle as part of their contact details when submitting manuscripts, this will enable the Twitter handle to be included when tweeting about the paper. 
Another trend to improve transparent, unbiased reporting of scientific data is the publication of scientific protocols. We believe that the publication of selected neurosurgical protocols will support scientific quality and reproducibility in neurosurgery and therefore we have adapted accordingly. In addition to our regular sections, we are pleased to introduce a new section "Study Protocol." The instructions for authors in their abbreviated form can be found on the Springer website: https:// www.springer.com/medicine/surgery/journal/701. The full instructions for authors have been placed on the editorial office website: www.di-ep.com

If you are interested in submitting a study protocol, you may either submit it directly or contact the editorial office beforehand for advice.

In the first quarter of this year, we have received almost 500 submissions, which is a clear increase from the first quarter of 2018. The quality of our submissions is continuously improving, and we have had Editorials on controversial topics that support debate and communication. We hope that by the end of the year we will have increased the scientific impact and number of submissions to our journal even more. Although the journal is the official European Journal of Neurosurgery, we welcome submissions from other non-European countries too.

We will be at the publication forum at the EANS 2019 Congress in Dublin and look forward to meeting you there. If you are on Twitter, do not forget to follow @ ActaNeuro; if not, perhaps use the fact that the journal is now on Twitter as an excuse and sign up (https://twitter.com/ActaNeuro?s=03).

\section{Compliance with ethical standards}

Conflict of interest There are no conflicts of interest to declare. Diana Epstein is the Managing Editor (Editorial Office), Angelos Kolias is the Team Leader for Social Media, and Tiit Mathiesen is the Editor-in-Chief.

\section{Appendix}

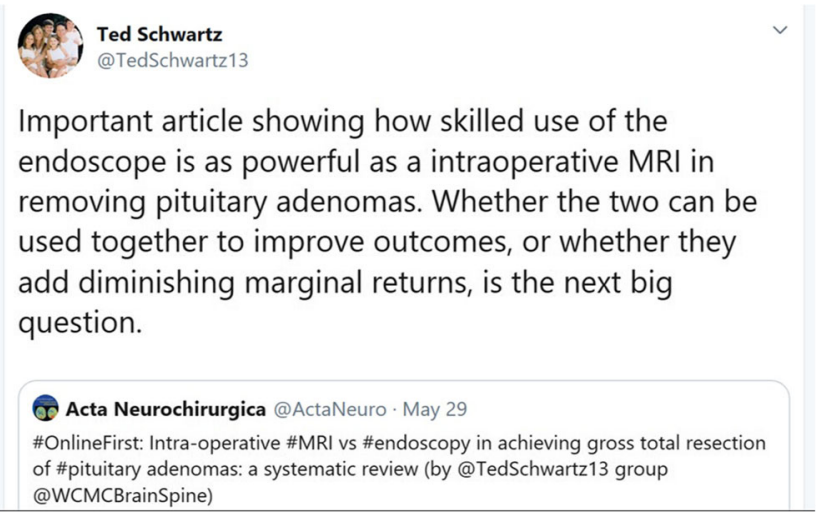

Fig. 2 Screenshot of a Tweet by Dr. Ted Schwartz (@TedSchwartz13) commenting on one of his papers

\section{Kee Park}

@keepa

Honored to be part of the diverse consensus group with a strong representation by \#TBI experts in the \#LMICS context.

6. Acta Neurochirurgica @ActaNeuro·May 28

\#OnlineFirst and \#OpenAccess: Consensus statement from the International \#Consensus Meeting on the Role of \#Decompressive \#Craniectomy in the Management of \#TBI by@WFNSHQ@global_neuro@GlobalNeuroOrg

\#neurosurgery \#nsgy \#neurotrauma

link.springer.com/article/10.100..

Fig. 3 Screenshot of a Tweet by Dr. Kee Park (@keepark) commenting on one of his papers

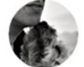

Simon Cudlip

@ukneurosurgeon

I've used the cross- court contralateral approach via a brow incision for the last few years for this very reason \#neurosurgery@ActaNeuro \#minisupraorbital

ब Acta Neurochirurgica@ActaNeuro.May 27

\#OnlineFirst: The approach angle to the interoptic triangle limits surgical workspace when targeting the contralateral internal carotid artery

\#neurosurgery \#nsgy \#skullbase \#ActaNeuro

link.springer.com/article/10.100...

Fig. 4 Screenshot of a Tweet by Dr. Simon Cudlip (@ukneurosurgeon) commenting on a paper about the approach angle to the interoptic triangle

\section{Reference}

1. Huang W, Wang P, Wu Q (2018) A correlation comparison between Altmetric Attention Scores and citations for six PLOS journals. PLoSONE 13(4):e0194962. https://doi.org/10.1371/journal.pone. 0194962

Publisher's note Springer Nature remains neutral with regard to jurisdictional claims in published maps and institutional affiliations. 